\title{
When warrant transmits and when it doesn't: towards a general framework
}

\section{Luca Moretti Tommaso Piazza}

\begin{abstract}
In this paper we focus on transmission and failure of transmission of warrant. We identify three individually necessary and jointly sufficient conditions for transmission of warrant, and we show that their satisfaction grounds a number of interesting epistemic phenomena that have not been sufficiently appreciated in the literature. We then scrutinise Wright's analysis of transmission failure and improve on extant readings of it. Nonetheless, we present a Bayesian counterexample that shows that Wright's analysis is partially incoherent with our analysis of warrant transmission and prima facie defective. We conclude exploring three alternative lines of reply: developing a more satisfactory account of transmission failure, which we outline; dismissing the Bayesian counterexample by rejecting some of its assumptions; reinterpreting Wright's analysis to make it immune to the counterexample.
\end{abstract}

Keywords Transmission of warrant; transmission failure; epistemic transmission; propositional warrant; Crispin Wright

\section{Introduction}

It is a philosophical commonplace that while arguing for a claim, one should not offer a valid argument whose premises explicitly include that very claim. Any such argument would be blatantly circular: it would be incapable of moving anyone to rationally accept its conclusion, as one should already accept its conclusion before going through the argument. In a series of publications Crispin Wright (1985, 2002, 2003, 2004, 2007 and 2011) has proposed an interesting analysis of epistemic circularity. According to Wright, epistemic circularity is generally instantiated when the warrant for the premises of a deductively valid argument is unable to transmit to its conclusion. Every circular argument of the type described above exhibits this flaw in a trivial way. Wright suggests, however, that the same flaw can be instantiated in a subtler and philosophically more interesting way by deductive arguments whose conclusion is not also a 
premise. ${ }^{1}$ Wright has analysed the general notion of transmission of warrant across known entailment and has proposed conditions that would suffice for non-trivial failure of transmission. Inspired by Wright's seminal papers, the recent literature has focused on transmission and failure of transmission of warrant. ${ }^{2}$ Despite this interest, there has been almost no attempt to develop a comprehensive analysis of the various ways in which these two elusive epistemological phenomena can obtain. ${ }^{3}$ This paper aims to provide at least the outline of such a comprehensive picture.

We kick off in $\S 2$ elucidating what transmission of warrant across known entailment is. We identify three conditions whose joint satisfaction appears sufficient for transmission of warrant. In $\S 3$ we pause to investigate why warrant transmission is an important epistemic phenomenon. While the current literature mainly focus on transmission of what has been called first time warrant, we lay down a more comprehensive taxonomy of types of transmissible warrant and briefly illustrate epistemic benefits of transmission of warrant of each type. In $\S 4$ we dissect Wright's account of transmission failure, improving on extant interpretations of it. We identify two independent conditions that Wright seems to consider sufficient for transmission failure, and we use a Bayesian counterexample to show that the more interesting condition is defective or, at the very least, cannot be regarded as completely satisfactory as it stands. In $\S 5$ we examine three alternative lines of reply: developing a more adequate account of transmission failure, which we outline; dismissing the Bayesian counterexample by rejecting its assumptions; defending a reinterpretation of Wright's analysis that would render it immune to the counterexample. In $\S 6$ we draw our conclusions.

\footnotetext{
${ }^{1}$ An important case in point would be, according to Wright (2002), Moore's proof of a material world.

${ }^{2}$ See for instance Moretti (2011), Chandler (2010), Tucker (2010), Smith (2009), Silins (2005), Ebert (2005) and Okasha (2004).

${ }^{3}$ The only exception seems to be Wright (2011), which sketches an interesting but quite "compressed" taxonomy. We are indebted with a referee of this Journal for drawing our attention to this very recent paper.
} 


\section{When warrant transmits}

Wright distinguishes transmission of warrant across known entailment from closure of warrant under known entailment. The closure principle says that "whenever there is warrant for the premises of a (known) valid argument, there is warrant for the conclusion too" (Wright 2002: 332). Wright does not question the validity of this principle, and we will follow him in not doing it. The transmission principle is more specific than the closure principle; it says that: "to acquire a warrant for the premises of a valid argument and to recognise its validity is thereby to acquire perhaps for the first time - a warrant to accept the conclusion" (ibid.). We will see that to acquire a warrant for the premises of a valid argument and to recognise its validity doesn't always result in acquiring a warrant for its conclusion. Thus the transmission principle is not generally valid. ${ }^{4}$

Wright's neatest characterisation of the notion of warrant transmissibility is possibly this:

A warrant is transmissible [across a valid argument] when we may envisage a logically nonomniscient but otherwise perfectly rational subject coming to believe [its conclusion] for the first time in a way which depends on their recognising both the validity of the [argument] in question and that they possess a warrant for its premises.

(Ibid.).

Whether or not a warrant is transmissible normally depends on background information (cf.

Wright 2003: 58). First of all, a perfectly rational subject $S$ will recognise that her evidence does warrant a proposition - typically - taking into account her background information. Furthermore, for Wright, a valid argument from P to Q is not necessarily one in which Q logically follows from just $\mathrm{P}$; a valid argument is also one in which $\mathrm{Q}$ logically follows from $\mathrm{P}$ only in conjunction with

\footnotetext{
${ }^{4}$ Wright analyses transmission and failure of transmission of both evidential and non-evidential warrant (see for instance Wright 2002 and 2011). According to Wright there is non-evidential warrant for P if P's truth is apprehended directly by the operation of some suitable cognitive faculty - e.g. perception conceived as a form of non-inferential awareness of external facts. To keep our paper manageable we will confine our analyses to transmission and failure of transmission of evidential warrant only. Whenever we say that a subject acquires a warrant for believing a premise $\mathrm{P}$, we will always mean that the subject's acceptance of a proposition (e.g. an observation report) E gives her defeasible justification for believing P. For a defence of a propositional conception of evidence see Williamson (2000).
} 
background information. Thus, $S$ may often recognise that a proposition entails another proposition only considering her background information. ${ }^{5} \mathrm{We}$ will follow Wright in using "validity" and "entailment" in these loosened senses.

Here is one of Wright's examples of warrant transmission:

\section{(Toadstool)}

E. Three hours ago, Jones inadvertently consumed a large risotto of Boletus Satana.

P. Jones has absorbed a lethal quantity of the toxins that toadstool contains.

Therefore:

Q. Jones will shortly die.

(2002: 332).

Here $\mathrm{E}$ does warrant $\mathrm{P}$ only given background information including assumptions about the toadstools in the risotto (e.g. that that specific variety of Boletus Satana is actually rich in lethal toxins). Furthermore, $\mathrm{P}$ is known to entail Q only in conjunction with background information including scientific assumptions (e.g. that a lethal quantity of those toxins will kill an ordinary human being shortly after its absorption) and assumptions about Jones (e.g. that Jones is an ordinary individual). In this example it appears true that a logically non-omniscient but perfectly rational subject $S$ (provided with suitable background information) would come to believe Q for the first time in a way that depends on her recognising that $\mathrm{E}$ warrants $\mathrm{P}$ and that $\mathrm{P}$ entails $\mathrm{Q}$.

In his analysis of warrant transmission Wright refers to the doxastic warrant that a perfectly rational subject $S$ would possess in a given epistemic situation to speak of the corresponding propositional warrant that we (non-ideally rational beings) would possess in the same situation. ${ }^{6}$ Wright's plausible assumption seems to be that whenever $S$ and we occupy the same epistemic

\footnotetext{
${ }^{5}$ A referee of this Journal has drawn our attention to the potential confusion between $S$ being able to know that $\mathrm{P}$ alone entails $\mathrm{Q}$ only against her background information, and $S$ being able to know that $\mathrm{P}$ together with her background information entails Q. Throughout the paper, when we say that $S$ can know that P entails Q (or that P is known to entail Q) only in conjunction with background information, we always mean this in the second sense.

${ }^{6}$ A subject has propositional warrant to believe a proposition $\mathrm{P}$ just in case, whether or not she actually believes $\mathrm{P}$, it would be epistemically appropriate for her to believe P. On the other hand, a subject is doxastically warranted in believing $\mathrm{P}$ just in case she has propositional warrant for believing $\mathrm{P}$ and she actually believes $\mathrm{P}$ in virtue of that warrant.
} 
situation (i.e. have the same information), what $S$ does believe coincides with what we should believe - namely, with what is propositionally warranted for us. Hence, what would be doxastically warranted for $S$ in a given situation can be used to model what is propositionally warranted for us in the same situation. Paralleling this reasoning, what would not be doxastically warranted for $S$ in a given situation can be used to model what is not propositionally warranted for us in the same situation.

Within this model it is to be expected that if $S$ is doxastically warranted in believing $\mathrm{Q}$, and this fact depends on $S$ 's recognising that $\mathrm{E}$ does warrant $\mathrm{P}$ and that $\mathrm{P}$ entails $\mathrm{Q}$, the corresponding propositional warrant for Q depends on the propositional warrant from $\mathrm{E}$ for $\mathrm{P}$ and the known entailment from $\mathrm{P}$ to Q. Let us clarify the features of this relation of dependence in play both at doxastic and propositional level. This is a natural way to construe the relation of dependence at doxastic level:

(a) If $S$ did not recognise that her evidence $\mathrm{E}$ does warrant $\mathrm{P}$ or did not recognise that $\mathrm{P}$ entails Q, $S$ would acquire no first-time warrant for Q.

Since $S$ is a logically non-omniscient but ideally rational thinker, it seems a priori true that $S$ does not recognise that her evidence $\mathrm{E}$ supplies a warrant for $\mathrm{P}$ against her background information if and only if $\mathrm{E}$ does not supply a propositional warrant for $\mathrm{P}$ against that background information. Furthermore, it is a priori true that $S$ does not recognise that $\mathrm{P}$ entails $\mathrm{Q}$ against her background information if and only if $\mathrm{P}$ is not actually known to entail $\mathrm{Q}$ against that background information. ${ }^{7}$ Finally, it appears a priori true that $S$ acquires no first-time warrant for Q upon learning E given her background information if and only if acquiring E provides no first-time propositional warrant for Q against that background information. We can thus turn (a) into (b):

\footnotetext{
${ }^{7}$ We can stipulate that $S$ 's logical ability is the same as that of a typical member of our linguistic community.
} 
(b) If E did not supply a warrant for P or P were not known to entail Q, there would be no first-time warrant for $\mathrm{Q}^{8}{ }^{8}$

Condition (b) encapsulates the relation of dependence we were after at propositional level. Let us test (b) on Toadstool. Assume that E does not warrant P or that P is not known to entail Q because our background information has been slightly $\operatorname{modified}^{9}$ (e.g. it now says that the specific variety of Boletus Satana in Jones' risotto is not rich in lethal toxins, or that Jones' response to the lethal toxins is extraordinarily slow). In these cases it is intuitive that there would be no propositional warrant for Q, as expected.

Though (b) would seem to reproduce at propositional level the dependence condition necessary for warrant transmission described in Wright's above quotation, we are not fully satisfied with (b) as long as (b) is meant to be a general condition for warrant transmission. As we will shortly clarify, the warrant from E for P can be transmitted to P's known consequence Q even if Q was already warrantedly believable on mere background information before the acquisition of $\mathrm{E}$ and the deduction of Q from P. In this case - to remain in line with Wright's general approach - an ideally rational thinker $S$ who already warrantedly believed Q could acquire a new warrant for Q in a way that depends on her recognising that $\mathrm{E}$ warrants $\mathrm{P}$ and that $\mathrm{P}$ entails $\mathrm{Q}$. This dependence condition at doxastic level can be fleshed out as follows:

(c) If $S$ did not recognise that $\mathrm{E}$ does warrant $\mathrm{P}$ or that $\mathrm{P}$ entails $\mathrm{Q}, S$ would acquire no new warrant for Q.

\footnotetext{
${ }^{8}$ Tucker (2010: 506) distinguishes between propositional warrant and propositional justification. While propositional warrant is a monadic property (e.g. $\mathrm{X}$ is propositionally warranted), propositional justification is a dyadic relation (X is propositionally justified by Y). Following Silins (2005), Tucker suggests that while propositional warrant can be transmitted across the entailment from $\mathrm{P}$ to $\mathrm{Q}$, propositional justification typically cannot. The reason would be that whereas the propositional justification for $\mathrm{P}$ is $\mathrm{E}$, the propositional justification for $\mathrm{Q}$ is, not $\mathrm{E}$, but $\mathrm{P}$ itself. We do not take a position on Tucker's suggestion. It is worth noting that (b) does not involve that a transmissive argument transmits propositional justification. In light of Tucker's distinction, (b) can be interpreted as a condition for transmission of propositional warrant. In general, whenever we say that the warrant from E for P transmits to P's consequence $\mathrm{Q}$, we just mean that $\mathrm{Q}$ is propositionally warranted in virtue of $\mathrm{P}$ 's being propositionally warranted. This is compatible with $\mathrm{Q}$ being propositionally justified by $\mathrm{P}$, and $\mathrm{P}$ being propositionally justified by $\mathrm{E}$.

${ }^{9}$ We use Lewis-Stalnaker semantics for counterfactuals: a counterfactual is true in a world $w$ just in case in the closest possible worlds to $w$ where its antecedent is true, its consequent is also true.
} 
The propositional version of $(\mathrm{c})$ is:

(d) If E did not warrant P or P were not known entail Q, there would be no new warrant for Q.

The obvious way to merge (b) with (d) is this:

(DEPENDENCE) If $\mathrm{E}$ did not warrant $\mathrm{P}$ or $\mathrm{P}$ were not known to entail $\mathrm{Q}$, there would be no first-time warrant for $\mathrm{Q}$ (if $\mathrm{Q}$ is not antecedently warranted) or no new warrant for $\mathrm{Q}$ (if $\mathrm{Q}$ is antecedently warranted).

As there can be warrant transmission only if the warrant for $\mathrm{Q}$ depends on the warrant from $\mathrm{E}$ for $\mathrm{P}$ and on the known entailment from $\mathrm{P}$ to $\mathrm{Q}$, DEPENDENCE appears to be a necessary condition for warrant transmission.

What other conditions are necessary for warrant transmission? Consider again Wright's general characterisation of warrant transmissibility quoted above. Trivially, if available evidence E did not warrant $\mathrm{P}$ or if $\mathrm{P}$ were not known to entail $\mathrm{Q}$, the envisaged rational thinker $S$ could not recognise that $\mathrm{E}$ warrants $\mathrm{P}$ and that $\mathrm{P}$ entails $\mathrm{Q}$. Thus transmission of warrant requires another condition to be satisfied:

(P-WARRANT) E does warrant $\mathrm{P}$, and $\mathrm{P}$ is known to entail Q.

Finally, $S$ could not come to believe Q via transmission if she did not acquire a warrant for Q upon recognising that that $\mathrm{E}$ warrants $\mathrm{P}$ and that $\mathrm{P}$ entails $\mathrm{Q}$. Transmission of warrant requires thus a third necessary condition to be satisfied that roughly says that Q must receive a warrant. Let us call this condition Q-WARRANT.

Though it is trivial that Q-WARRANT must be satisfied in case of transmission, to specify what this condition exactly imposes is all but trivial. Wright states - in the passage quoted above that the warrant from $\mathrm{E}$ for $\mathrm{P}$ transmits to P's consequence $\mathrm{Q}$ only if we can envisage an ideally 
rational thinker coming to believe Q for the first time. Wright mostly concentrates on transmission of first-time warrant: i.e. on cases in which belief in Q was not antecedently warranted before the acquisition of $\mathrm{E}$, so that the warrant transmitted to $\mathrm{Q}$ is a first-time warrant for it. These examples include Toadstool. It seems natural, however, that one should accept as cases of transmission also those in which the warrant from P to Q is not first-time but one that would have been so if Q had not already been warranted. These are cases of transmission of a new counterfactually independent warrant. Wright's notion of warrant transmission embraces these cases too. This can be evinced, for example, from Wright's formulation of the principle of transmission of warrant quoted at the beginning of this section if "perhaps" is interpreted as "possibly". ${ }^{10}$ (Cf. also Wright 2011: 83).

More accurately, a warrant for Q is new and counterfactually independent of Q's antecedent warrant just in case the following conditions are satisfied: (i) Q is already warranted on mere background information, (ii) Q remains warranted when E is acquired, and (iii) if Q had not been antecedently warranted, Q would have been first-time warranted upon acquiring E. The following example will help to clarify this notion. Suppose you are travelling from London to Edinburgh and you have been sleeping for a while. At 16:00 the train stops, and you just wake up. You see that the station is Newcastle upon Tyne. So at 16:00 you have a warrant for believing that (Q) you are not yet in Edinburgh. Consider now this reasoning:

(Journey)

E. At 16:05 the ticket controller tells you that you are not yet in Scotland.

P. You are not yet in Scotland.

Therefore:

Q. You are not yet in Edinburgh.

\footnotetext{
${ }^{10}$ On this interpretation that principle says: to acquire a warrant for the premises of a valid argument and to recognise its validity is thereby to acquire - possibly for the first time - a warrant to accept the conclusion.
} 
As you learn E, it is intuitive that you acquire a warrant for Q via transmission - i.e. a new counterfactually independent warrant. This is so because (i) Q was already warranted for you. Besides, (ii) as you acquire E, you remain warranted in believing Q. Furthermore, it is true that (iii) if Q had not been antecedently warranted (e.g. if you had not spotted the station's name), you would have received a first-time warrant for Q upon acquiring E. For E would have warranted your belief in $\mathrm{P}$, which you knows entails $\mathrm{Q}$.

There is at least another intuitive sense in which a new warrant can transmit across known entailment. For Wright, if E supplies a warrant for X, E makes X rationally believable or acceptable. Wright recognises that warrant comes typically in degrees of strength; ${ }^{11}$ so when he says that E warrants $\mathrm{X}$, he presumably means that the strength of the warrant from $\mathrm{E}$ for $\mathrm{X}$ is sufficient to make $\mathrm{X}$ believable or acceptable. On this conception, a warrant already sufficient for believing a proposition can further be strengthened, in the sense that its degree can further be raised if new evidence is acquired. This suggests the novel sense in which a new warrant can transmit. Note that in Journey when E is acquired, Q remains warranted because it is intuitive that the degree of Q's warrant does not change. Below we give an example of transmission in which Q remains warranted though its degree of warrant decreases. Yet there are cases of transmission in which when $\mathrm{E}$ is acquired, Q remains warranted because its degree of warrant increases. In these cases it is quite natural to conclude that a new quantitatively stronger warrant transmits to Q. More precisely, a warrant transmitted from $\mathrm{P}$ to its known consequence $\mathrm{Q}$ is a new warrant quantitatively stronger than Q's antecedent warrant just in case the following conditions are satisfied: Q is already warranted on mere background information, and the degree of Q's warrant

\footnotetext{
$\overline{{ }^{11} \text { See for instance Wright (2007: 36). }}$
} 
raises as $\mathrm{E}$ is acquired. Wright (2011: 83-84) seems to recognise that this is one of the senses in which warrant can transmit. ${ }^{12}$

Here is an example from Moretti (2011). Suppose your background information says that only one ticket out of 5,000 of a fair lottery has been bought by someone born in 1970, and that all other tickets have been bought by older or younger people. The proposition that (Q) the lottery winner was not born in 1970, given its high chance, is already warranted on your background information only. Consider now this reasoning:

(Lottery)

E. The lottery winner's passport certifies she was born in 1980.

P. The lottery winner was born in 1980 .

Therefore:

Q. The lottery winner was not born in 1970 .

As you learn E, it is intuitive that you acquire a warrant for Q via transmission - i.e. a new quantitatively stronger warrant for $\mathrm{Q}$. This is so because $\mathrm{Q}$ was already warranted for you. Furthermore, E provides you with a very strong warrant for $\mathrm{P}$ - intuitively, one quantitatively stronger than your initial warrant for $\mathrm{Q}$. Since you know that $\mathrm{P}$ entails $\mathrm{Q}$, the degree of your warrant for Q cannot presumably be weaker than the degree of your warrant for P. Hence, your final warrant for $\mathrm{Q}$ will be quantitatively stronger than your initial warrant for $\mathrm{Q}$.

In light of the above considerations we propose to formulate Q-WARRANT as the following tripartite disjunctive condition:

(Q-W1) Q was not antecedently warranted and there is a first-time warrant for Q;

or

(Q-W2) There is a new warrant for Q counterfactually independent of Q's existing antecedent warrant;

or

(Q-W3) There is a new warrant for Q quantitatively stronger than Q's existing antecedent warrant.

\footnotetext{
${ }^{12}$ Chandler (2010) also recognises this sense of warrant transmission because his Bayesian model aims to account for transmission of both first-time warrant and new quantitatively stronger warrant.
} 
More generally, it seems to us that the notion of transmission of warrant can be couched in terms of the following three conditions:

(P-WARRANT) E does warrant $\mathrm{P}$, and $\mathrm{P}$ is known to entail $\mathrm{Q}$.

(Q-WARRANT) Q-W1 or Q-W2 or Q-W3.

(DEPENDENCE) If E did not warrant $\mathrm{P}$ or $\mathrm{P}$ were not known to entail $\mathrm{Q}$, there would be no first-time warrant for $\mathrm{Q}$ or no new warrant for $\mathrm{Q}$ (respectively according to the satisfaction of Q-WARRANT).

We think that these three conditions are jointly sufficient for warrant transmission. It is easy to verify that all of them are satisfied - for instance - in Journey. ${ }^{13}$ P-WARRANT is fulfilled in Journey because E (“At 16:05 the ticket controller tells you that you are not yet in Scotland") supplies a warrant for P ("You are not yet in Scotland"), and P is known to entail Q ("You are not yet in Edinburgh"). Q-WARRANT is satisfied because, as we have seen, there is a new warrant for Q independent of Q's prior warrant. DEPENDENCE is satisfied because if E did not warrant $\mathrm{P}$ (e.g. if you knew that the controller is lying to you) or if $\mathrm{P}$ were not known to entail Q (i.e. if you did not know this entailment), there would be no new warrant for Q counterfactually independent of Q's antecedent warrant. ${ }^{14}$

We believe that P-WARRANT, Q-WARRANT and DEPENDENCE are also necessary for warrant transmission. In particular it seems to us that Q-W1, Q-W2 and Q-W3 exhaust the intuitive senses in which a warrant can be said to transmit to a proposition Q; if there are other senses, those accounted for by Q-WARRANT are presumably the principal. ${ }^{15}$ One might wonder however whether Q-WARRANT could be simplified. Journey seems to show that satisfying QW2 does not imply satisfying Q-W3 - namely, that transmitting to Q a new warrant

\footnotetext{
${ }^{13}$ The reader can verify that this is so also true of Toadstool and Lottery.

${ }^{14}$ More specifically, in either case the clause (iii) of the characterisation of new and independent warrant would remain unfulfilled, as it would be false that if you had had no antecedent warrant for Q - e.g. if had not glimpsed the station's name - you would have had a first-time warrant for Q upon acquiring E.

${ }^{15}$ Wright (2011: 86) suggests that there is transmission of warrant from $\mathrm{P}$ to its logical consequence Q when the argument from $\mathrm{P}$ to $\mathrm{Q}$ "is used to rationally move past an open-minded stance towards" Q. We find this notion of transmission puzzling and, in so far as we understand it, we suspect it is reducible to that accounted by Q-W1.
} 
counterfactually independent of Q's antecedent warrant does not entail transmitting to Q a new warrant quantitatively stronger than Q's antecedent warrant. ${ }^{16}$ Yet the only example of transmission satisfying Q-W3 that we have supplied - i.e. Lottery - is a case in which Q-W2 is also satisfied. One might thus suspect that every case of transmission in which Q-W3 is satisfied is one in which Q-W2 is fulfilled. If this were true, Q-WARRANT could be simplified into the disjunction of Q-W1 and Q-W2 alone. To dispel this suspect we now show that if warrant is construed as sufficiently high rational credence (or subjective probability), there are cases of transmission that satisfy Q-W3 but not $\mathrm{Q}-\mathrm{W} 2 .^{17}$

Suppose you participate in a psychology test (its ultimate purpose is irrelevant) involving a tool called the 3B-apparatus. Each 3B-apparatus is made of three boxes $A, B$, and $C$. Each box contains 9 black balls. Furthermore:

Box $A$ also contains 1 red ball, 10 white balls and nothing else. Only the red ball and 5 white balls are heavy.

Box $B$ also contains 7 red balls, 4 white balls and nothing else. Only 3 red balls and 3 white balls are heavy.

Box $C$ also contains 7 red balls, 4 white balls and nothing else. Only 5 red balls and 1 white ball are heavy.

\footnotetext{
${ }^{16}$ We will give another example perhaps more convincing in the next section.

${ }^{17}$ Wright (2011, pp. 83-84) provides a non-probabilistic example to support the same conclusion that we do not find persuasive. Suppose you see a rather mobile flock of sheep in a pen, and you are initially told that (Q) the sheep are fewer than twenty. Suppose also that (E) on counting as carefully as you can, you get the result eighteen. According to Wright, you could take E as intensifying your antecedent warrant for Q via transmission. If this is true, Q-W3 is satisfied. This would be your reasoning:

E. I counted the sheep as carefully as I could and got the result 18 .

P. There are 18 sheep in the pen.

Q. There are fewer than 20 sheep in the pen.

Wright suggests that in this case if you had lacked the antecedent warrant for Q, you could not have taken E to provide sufficient warrant to conclude that Q. This is why Q-W2 is not satisfied. We do not find this ingenious example convincing because we think that one might reasonably doubt that Q-W3 is fulfilled here. One could contend that whether or not $\mathrm{Q}$ is antecedently warranted, since the flock is rather mobile, $\mathrm{E}$ is insufficient to warrant $\mathrm{P}$.
} 
The test requires you to go through the following steps: you are first given the above information about your 3B-apparatus (and it is checked that you correctly remember it). You are then blindfolded and asked to carry out the following course of actions: first, you randomly select one of the boxes of your 3B-apparatus. After being told which box you have picked out, you randomly extract one ball from that box. You are asked to pronounce on whether that ball is (indifferently) black or red given its weight and your information about the selected box.

$$
\begin{aligned}
& \mathrm{E}=\text { "The extracted ball is heavy". } \\
& \mathrm{P}=\text { "The extracted ball is red". } \\
& \mathrm{Q}=\text { "The extracted ball is black or red". }
\end{aligned}
$$

As said, Wright recognises that warrant comes typically in degrees, and he seems to think that there is warrant to believe a proposition $\mathrm{X}$ if and only if the degree of warrant for $\mathrm{X}$ is sufficiently high. It is usual to model degrees of warrant (or justification) as degrees of rational credence or subjective probability. Let us assume therefore that there exists a threshold value $t$ for the degree of rational credence in a proposition $\mathrm{X}$ the exceeding of which results in possessing a warrant for believing $X{ }^{18}$ Imagine $t=3 / 4$. Then belief in $\mathrm{X}$ is warranted just in case $\operatorname{Pr}(\mathrm{X})>t=3 / 4$, where $\operatorname{Pr}(\mathrm{X})$ gives a subject's degree of rational credence in $\mathrm{X}$. Furthermore, $\mathrm{Y}$ is a warrant for believing $\mathrm{X}$ just in case $\operatorname{Pr}_{\mathrm{Y}}(\mathrm{X})>t=3 / 4$, where $\operatorname{Pr}_{\mathrm{Y}}(\mathrm{X})$ gives a subject's degree of rational credence in $\mathrm{X}$ upon her learning Y.

You begin the test. You randomly select one of your 3B-apparatus' boxes and you are told that that box is $C$. Given your initial information, you now know that the chance of Q is $4 / 5$. So it is natural to conclude that $\operatorname{Pr}(\mathrm{Q})=4 / 5$. Note that $\operatorname{Pr}(\mathrm{Q})>t$. As you are told that the selected box is $C$, you realise that the chance of $\mathrm{P}$ is $7 / 20$; thus $\operatorname{Pr}(\mathrm{P})=7 / 20$. Note that $\operatorname{Pr}(\mathrm{P})<t$. While keeping blindfolded, you then extract a ball from $C$ and acquire evidence E. E raises your degree of

\footnotetext{
${ }^{18}$ In doing this we follow Chandler (2010) and Moretti (2011).
} 
credence in $\mathrm{P}$ (given your background information) above $t$ because $\operatorname{Pr}_{\mathrm{E}}(\mathrm{P})=5 / 6>t$. E also raises your degree of credence in $Q$ (given your background information) because $\operatorname{Pr}_{E}(Q)=5 / 6>\operatorname{Pr}(Q)$ $=4 / 5$. Suppose that after extracting the ball from $C$, you reason as follows to judge whether that ball is black or red:

(Balls)

E. The extracted ball is heavy.

$P$. The extracted ball is red.

Therefore:

Q. The extracted ball is black or red.

Balls appears to be a case of transmission of new warrant; in particular, the warrant from E for P transmits to Q in form of a new warrant quantitatively stronger than Q's antecedent warrant. Yet in Balls no new warrant counterfactually independent of Q's antecedent warrant transmits to Q. So this is a case of transmission in which only Q-W3 but not Q-W2 is satisfied - this offers us the counterexample we have been looking for. Let us first show that P-WARRANT, Q-W3 and DEPENDENCE are all satisfied, so that transmission of a new quantitatively stronger warrant is instantiated. After this, we will show that Q-W2 is not fulfilled so that no transmission of new counterfactually independent warrant is instantiated.

P-WARRANT is satisfied in Balls because $\operatorname{Pr}_{\mathrm{E}}(\mathrm{P})>t$ - thus $\mathrm{E}$ does warrant $\mathrm{P}-$ and $\mathrm{P}$ is known to entail Q. Q-W3 is fulfilled because $\operatorname{Pr}_{\mathrm{E}}(\mathrm{Q})>\operatorname{Pr}(\mathrm{Q})>t$ - hence there is a new warrant for $\mathrm{Q}$ quantitatively stronger than Q's antecedent warrant. ${ }^{19}$ DEPENDENCE says, in this case, that if E did not warrant $\mathrm{P}$ or $\mathrm{P}$ were not known to entail $\mathrm{Q}$, there would be no new quantitatively stronger warrant for Q. This counterfactual is intuitively true in Balls. For the closest possible worlds in which $\mathrm{E}$ does not warrant $\mathrm{P}$ or $\mathrm{P}$ is not known to entail $\mathrm{Q}$ are presumably those in which the only

\footnotetext{
${ }^{19}$ Tucker (2010) claims that the ground of Q's warrant in case of transmission is, not E, but P. Tucker must mean that the ground of Q's warrant is $\mathrm{P}$ once $P$ has been warranted by $E$. Since the degree of warrant transmitted to Q depends ultimately on $\mathrm{E}$ on this view, $\operatorname{Pr}_{\mathrm{E}}(\mathrm{Q})$ could arguably be taken to measure the degree of the warrant transmitted to $\mathrm{Q}$ also in Tucker's sense.
} 
changes depend on your being told that you have randomly selected box $B$ rather than box $C$. In these worlds, on your slightly changed background information, $\operatorname{Pr}(\mathrm{Q})=4 / 5>t$. So you still have antecedent warrant for believing Q. However, $\operatorname{Pr}_{\mathrm{E}}(\mathrm{P})=1 / 2<t$, so E gives you no warrant for $\mathrm{P}$, and $\operatorname{Pr}_{\mathrm{E}}(\mathrm{Q})=1 / 2<t$, so you have no new warrant for Q quantitatively stronger than Q's antecedent warrant. Since in the closest possible worlds where DEPENDENCE's antecedent is true its consequent is also true, DEPENDENCE is satisfied.

Let us turn to Q-W2. The latter entails the counterfactual that if Q had not been antecedently warranted, Q would have been first-time warranted upon the acquisition of E. Q-W2 is not satisfied in Balls because this counterfactual is intuitively false. The closest possible worlds in which Q is not antecedently warranted are presumably those in which all changes depend on your being told that you have randomly selected box $A$ rather than $C$. In these worlds, on your slightly changed background information, $\operatorname{Pr}(\mathrm{Q})=1 / 2<t$, so $\mathrm{Q}$ is no longer antecedently warranted for you. However, $\operatorname{Pr}_{\mathrm{E}}(\mathrm{Q})=1 / 6<t$. Hence, you acquire no first-time warrant for $\mathrm{Q}$ as you learn $\mathrm{E}$. In conclusion, it is false that if Q had not been antecedently warranted, Q would have been first-time warranted upon acquiring E. As the counterfactual entailed by Q-W2 is false, Q-W2 is unsatisfied.

Balls gives strong evidence that fulfilling Q-W3 does not require fulfilling Q-W2 in case of transmission. Arguably, Balls gives no conclusive proof of this because its modelling assumptions might be questioned. We will return to this issue in $\S 5$. Note at any rate that the counterexample offered by Balls does not depend on selecting just 3/4 as the threshold value $t$ for warranted belief. Any other $t \in[1 / 2,3 / 4)$ would do, and Balls could suitably be modified to satisfy any $t \in[3 / 4,1)$ one might propose.

\section{Varieties of transmitted warrant and their epistemic benefits}


It appears intuitively plausible to us that a deductive argument from $\mathrm{P}$ to $\mathrm{Q}$ is transmissive only if the argument together with the warrant $\mathrm{E}$ for $\mathrm{P}$ would allow an ideally rational subject $S$ to improve in some sense her overall epistemic position relative to Q. Sometimes this improvement obtains just because the degree of $S$ 's prior warrant for Q increases. This happens when a firsttime warrant transmits. In this case, the prior degree of Q's warrant must necessarily increase because $S$ can acquire a first-time warrant for Q via transmission only if $S$ 's warrant for Q before acquiring E was insufficient to sustain her belief in Q. Transmission of first-time warrant is crucial for our cognitive enterprise because it makes the advancement of knowledge (or of warranted belief) through deductive reasoning possible.

There are cases of transmission also when the warrant for Q on mere background information already suffices to sustain belief in Q. In these situations, when E is acquired, the degree of Q's warrant may further increase; this happens for instance in Lottery. Let us call the new warrant transmitted in these cases intensifying warrant. Transmission of intensifying warrant improves $S$ 's overall epistemic situation regarding $Q$ because it boosts the warrant that $S$ already has for $Q$.

Yet if $\mathrm{Q}$ is already warranted and the warrant for $\mathrm{P}$ transmits to $\mathrm{Q}$, the degree of the antecedent warrant for Q may remain unchanged or even decrease. ${ }^{20}$ In these cases, transmission of warrant is still valuable from an epistemic viewpoint. Consider for instance Journey. As you learn E (“At 16:05 the ticket controller tells you that you are not yet in Scotland"), though the degree of your antecedent warrant for Q ("You are not yet in Edinburgh") presumably remains unchanged, Q's epistemic standing relative to you improves nonetheless. For the new warrant transmitted to Q is counterfactually independent of the antecedent warrant for $\mathrm{Q}$, with the consequence that your

\footnotetext{
${ }^{20}$ Wright (2011: 84) makes the same point but he tends to conceive of improving $S$ 's overall epistemic position regarding Q as increasing $S$ 's prior credence in Q. Consequently, Wright does not explain how $S$ 's acquisition of warrant for Q via transmission improves $S$ 's overall epistemic position regarding Q when $S$ 's prior credence in Q does not rise.
} 
overall warrant for Q is now more stable: if the antecedent warrant for Q were defeated, in many cases the transmitted warrant would survive. Imagine for example that after learning E, you read on the newspaper that some hooligans have been enjoying putting signs with the name "Newcastle upon Tyne" in place of the signs with the name "Edinburgh" at Edinburgh railway station to confuse passengers. This defeats your initial warrant for Q. Yet you are still entitled to believe Q by the new warrant transmitted to $\mathrm{Q}$. Whenever transmission establishes a warrant stronger than before in this qualitative sense, we say that a ground-extending warrant is transmitted. Whether or not the ground of a warrant becomes more extended in this sense seems to be largely independent of the degree of the resulting warrant. ${ }^{21}$ We should thus not confuse this qualitative sense in which transmission may establish a stronger warrant with the quantitative (or intensifying) sense in which transmission may establish a stronger warrant. (Note however that a ground-extending warrant can simultaneously be intensifying).

It may happen that $\mathrm{Q}$ is already warranted by background information, and new evidence $\mathrm{E}$ supplies a warrant for P that transmits to Q as a new counterfactually independent warrant that defeats Q's antecedent warrant. In this case $S$ would improve her overall epistemic position about Q because $S$ would replace an ill-founded warrant for Q with a (prima face) trustworthy warrant for it. We call any transmitted warrant of this kind proxy warrant. Consider this example: since you spotted the obituary of Andrea in the yesterday Times, you have background evidence $\mathrm{E}^{*}$ warranting the proposition (Q) that Andrea will not spend her holiday in Mexico. (E* could be the proposition that you have read the obituary). Consider now this reasoning:

(Holiday)

E. Unexpectedly, you meet Andrea in town; she says she will not leave Europe for holiday. P. Andrea will not leave Europe for holiday. Therefore:

\footnotetext{
${ }^{21}$ For a nice example in which the ground of a warrant is extended via transmission while its degree is reduced see Wright (2011: 84-85).
} 
Q. Andrea will not spend her holiday in Mexico.

E gives you a warrant for $\mathrm{P}$ but $\mathrm{E}$ also defeats your original warrant from $\mathrm{E}^{*}$ for $\mathrm{Q}$. As you deduce Q from $\mathrm{P}$, your warrant from $\mathrm{E}$ for $\mathrm{P}$ transfers to Q. So you still have warrant for believing Q. In this example it is intuitive that the final degree of warrant for Q is lower than the initial one. But this is just one possibility: it is easy to imagine cases in which the final degree of the warrant for $\mathrm{Q}$ is equal to or higher than the initial one. ${ }^{22}$

The moral that the discussion of this section buttresses is this: transmission of warrant from $\mathrm{P}$ to $\mathrm{Q}$ is valuable, not just because it raises the degree of the warrant for $\mathrm{Q}$ (as it appears presupposed in much of the current literature on the topic), but because it satisfies a number of different functions that improve the epistemic position of the subject with respect to Q, some of which have been illustrated by us in relation to first-time, intensifying, ground-extending and proxy warrant. Transmission across entailment can supply a first-time warrant for Q, or it can quantitatively or qualitatively reinforce, or "cleanse" and preserve an already possessed warrant for Q. We do not take this short list of epistemic benefits of warrant transmission to be exhaustive; further functions could probably be individuated. This is a reason why the phenomenon of warrant transmission is so intriguing and valuable.

\section{When warrant fails to transmit}

Although warrant often transmits across known entailment, there are cases in which it doesn't. Transmission failure across entailment from $\mathrm{P}$ to $\mathrm{Q}$ does not seem to obtain if $\mathrm{P}$ is de facto not warranted in the first place. Compare the case of signal transmission failure in a

\footnotetext{
${ }^{22}$ A possible useful function of proxy warrant is that of de-Gettierising the subject's epistemic situation. It is easy to imagine Gettier examples in which Q is true and $S$ 's belief in Q is warranted by $E^{*}$, which is yet ill-founded. Suppose then that $S$ 's learning E defeats $S$ 's misleading warrant from E* for Q and gives $S$ a warrant for P. As $S$ deduces Q from $\mathrm{P}$, the warrant from $\mathrm{E}$ for $\mathrm{P}$ transfers to $\mathrm{Q}$. This new warrant for $\mathrm{Q}$, unlike the old warrant, could sustain $S$ 's knowledge that $\mathrm{Q}$.
} 
telecommunications system: if there is no signal to transmit, there can be no failure of

transmission. Transmission failure is instantiated - rigorously speaking - only if there is warrant for $\mathrm{P}$ that does not transmit to $\mathrm{Q}$.

This is one of Wright's examples (cf. 2003: 59). Suppose Jessica and Jocelyn are known to be indistinguishable twins. The following reasoning, according to Wright, instantiates transmission failure:

(Twins)

E. This girl looks just like Jessica.

P. This girl is actually Jessica.

Therefore:

Q. This girl is not Jocelyn.

Here it is intuitive that the warrant from $\mathrm{E}$ for $\mathrm{P}$ does not transmit to Q. From examples like this, Wright abstracts this general condition that he believes to suffice for transmission failure:

Transmission may fail in a case when there is warrant for the premises in the first place only because the conclusion is antecedently warranted. (Wright 2002: 332; first emphasis ours).

Saying that the conclusion is antecedently warranted is equivalent, for Wright, to saying that the conclusion is already (and so) independently warranted. Twins instantiates this condition: if E warrants $\mathrm{P}$ in the first place, it can do so only because Q is already and independently warranted by background information. Given this, one could not acquire a warrant for believing Q via acquiring the warrant from $\mathrm{E}$ for $\mathrm{P}$ and deducing $\mathrm{Q}$ from $\mathrm{P}$. In another passage Wright states that warrant transmission fails "if the warrant for P supplied by E depends in the first place on prior and independent warrant for" $\mathrm{Q}$ (2002: 336; our emphasis). ${ }^{23}$

To fully grasp Wright's condition for failure of transmission it is crucial to comprehend what

\footnotetext{
${ }^{23}$ Wright (2011: 86) engages in "a little retrospective self-exegesis" by reinterpreting his account of transmission failure as specifically focusing on second-order warrant (i.e. warrant for claiming that one has warrant for believing a proposition) rather than first-order warrant (i.e. warrant for believing a proposition). Wright's self-exegesis seems not to have relevant implications for us. For it is intuitively plausible that on the notion of evidential warrant we have been presupposing in our analyses (see note 4 above), first-order and second-order warrant come typically together.
} 
Wright means when he says that $\mathrm{P}$ is warranted by $\mathrm{E}$ only because $\mathrm{Q}$ is antecedently warranted or - presumably equivalently for Wright - in a way that depends on antecedent warrant for Q.

Okasha (2004: 140) implicitly assumes that "only because" means "only if". Chandler (2010) and, with more reservation, Moretti (2011) tentatively construe Wright's condition by interpreting "only because" as, again, the material conditional. ${ }^{24}$ Let us dub this interpretation the OCMconstrual. We do not accept the OCM-construal because, so interpreted, Wright's analysis of transmission failure cannot be made to square with the conception of warrant transmission construed as in $\S 2$, which appears plausible in itself and that we believe Wright would substantially endorse. Furthermore, the OCM-construal is problematic on its own terms.

Let us first consider the latter point. Suppose P entails Q. The OCM-construal commits one to accepting the following propositions as equivalent:

(1) P is warranted only because Q is antecedently warranted.

(2) P is warranted only if $\mathrm{Q}$ is antecedently warranted.

Since "only if" is the material conditional, (2) is equivalent to:

$\left(2^{*}\right) \mathrm{P}$ is not warranted or $\mathrm{Q}$ is antecedently warranted.

Thus, if "only because" coincides with the material conditional, we have to regard every case in which the sole premise of a deduction is not warranted, or in which the conclusion is antecedently warranted, as a case in which the premise is warranted only because the conclusion is antecedently warranted.

Note, to begin with, that the truth of first disjunct of $\left(2^{*}\right)$ produces an odd effect. If $\mathrm{P}$ is not warranted, $\left(2^{*}\right)$ is true. But $\left(2^{*}\right)$ is equivalent to (2); hence if (2) is equivalent to (1), the latter claim is true too. Thus, if $\mathrm{P}$ is not warranted, we should claim that $\mathrm{P}$ is warranted only because Q is antecedently warranted, which appears false. One might perhaps disregard this odd

\footnotetext{
${ }^{24}$ This is one of the interpretations of Wright's condition suggested in Chandler (2010).
} 
consequence. Even so, other problems emerge as one considers the second disjunct of $(2 *)$. On this interpretation of "only because", every known deductive argument from $\mathrm{P}$ to $\mathrm{Q}$ in which $\mathrm{Q}$ is de facto antecedently warranted is an argument that verifies (1), i.e. one in which $\mathrm{P}$ is warranted only because Q is antecedently warranted. This appears, again, odd and false.

The latter upshot also shows that on the OCM-construal, Wright's sufficient condition for transmission failure is at odds with the conception of warrant transmission presented in $\S 2$. For if (1) is satisfied by every known deductive argument from $\mathrm{P}$ to $\mathrm{Q}$ in which $\mathrm{Q}$ is de facto antecedently warranted, then every known deductive argument from $\mathrm{P}$ to $\mathrm{Q}$ in which $\mathrm{Q}$ is de facto antecedently warranted is one in which the warrant for P is not transmissible to Q. If "only because" coincides with the material conditional, Wright's above quotation implies that transmission of new warrant (and so of intensifying, ${ }^{25}$ ground-extending and proxy warrant) is just impossible. Since all this is very implausible, we conclude that the OCM-interpretation of Wright's condition is too uncharitable to be accepted.

All problems discussed so far fade away if Wright's claim that E supplies a warrant for P only because there is antecedent warrant for Q (or in a way that depends on antecedent warrant for Q) is given a counterfactual interpretation. On this new reading, (1) is equivalent to:

(FAIL1) If there were no antecedent warrant for Q, E would not supply a warrant for P. ${ }^{26}$ If FAIL1 is Wright's condition for transmission failure, it is false that any known deductive argument in which Q is de facto antecedently warranted satisfies Wright's condition. In any case in which Q is de facto antecedently warranted but the warrant from $\mathrm{E}$ for $\mathrm{P}$ would survive if $\mathrm{Q}$ were not antecedently warranted, FAIL1 remains unsatisfied. In these cases warrant can transmit

\footnotetext{
${ }^{25}$ This problem has already been touched upon by Moretti (2011).

${ }^{26}$ This interpretation is also adumbrated in Chandler (2010).
} 
(this happens for instance in Journey). In conclusion FAIL1 would seem to reproduce the condition that Wright has in mind much more accurately than (2).

The crucial question is whether the fulfilment of FAIL1 suffices for transmission failure when the analysis of warrant transmission given in $\S 2$ is accepted. If FAIL1 is a sufficient condition for transmission failure, this must be the case because FAIL1 entails the impossibility of satisfying QWARRANT together with P-WARRANT and DEPENDENCE. Since Q-WARRANT is the disjunction of Q-W1, Q-W2 and Q-W3, this must be the case because FAIL1 entails the impossibility of satisfying either of these disjuncts together with P-WARRANT and DEPENDENCE. Let us test this claim.

To begin with, FAIL1 entails that Q-W1 is false. For FAIL1 says that if Q were not antecedently warranted $\mathrm{E}$ would not warrant $\mathrm{P}$, and a consequence of this is that there would be no first-time warrant for Q. This entails that if Q is not antecedently warranted, E does not warrant P, with the consequence that there is no first-time warrant for Q. In contrast, Q-W1 just says that Q is not antecedently warranted and there is first-time warrant for Q. Note also that FAIL1 is overtly incompatible with Q-W2. The latter says that there is a new warrant for Q counterfactually independent of Q's antecedent warrant. This entails, against FAIL1, that that if Q were not antecedently warranted, E would still warrant P.

A problem emerges as we turn to Q-W3. Wright (2007) briefly considers transmission of (what we have called) intensifying warrant, which requires the satisfaction of Q-W3. He suggests that intensifying warrant fails to transmit whenever his condition for transmission failure is satisfied: “the warrant for Q cannot in the circumstances [i.e. when the warrant for E for P depends on antecedent warrant for Q] be stronger than the warrant for $\mathrm{P}$, and the latter, presumably, is bounded by the strength of the demanded anterior warrant for Q" (ibid.: 36, slightly modified). In 
other words, Wright suggests that if the warrant from E for P depends on antecedent warrant for Q, the degree of the warrant for P cannot exceed the degree of the antecedent warrant for Q. For this reason - Wright claims - when his condition for transmission failure is satisfied, no warrant stronger than Q's antecedent warrant can transmit from P to Q. If Wright is correct and FAIL1 faithfully reproduces Wright's condition, FAIL1 must be a sufficient condition for transmission failure across the board. We should thus expect FAIL1 to entail that Q-W3 cannot be fulfilled together with P-WARRANT and DEPENDENCE. The problem is that Balls, presented in $\S 2$, can be used to show that FAIL1 can be satisfied when Q-W3, P-WARRANT and DEPENDENCE are jointly satisfied. ${ }^{27}$

In $\S 2$ we have argued that intensifying warrant does transmit in Balls (when warrant is identified with sufficiently high probability). We now argue that FAIL1 is also satisfied in Balls. FAIL1 says that if there were no antecedent warrant for Q, E would not warrant P. The closest possible worlds to the actual one (imagined in Balls) in which the antecedent of FAIL1 is true are those in which the only changes depend on your being told that you have randomly picked out box $A$ rather than $C$. In these worlds, $\operatorname{Pr}(\mathrm{Q})=1 / 2<t-$ i.e. $\mathrm{Q}$ is $n o t$ antecedently warranted - and

\footnotetext{
${ }^{27}$ In $\S 2$ we have used Balls to provide an example in which Q-W2 is not satisfied when Q-W3 is satisfied together PWARRANT and DEPENDENCE. Note that this example does not refute by itself the thesis that FAIL1 prevents the fulfilment of Q-W3 together P-WARRANT and DEPENDENCE. The latter thesis says that (to simplify let us disregard P-WARRANT and DEPENDENCE):
}

(a) IF the warrant from $\mathrm{E}$ for $\mathrm{P}$ counterfactually depends on antecedent warrant for Q, THEN the degree of the new warrant for $\mathrm{Q}$ cannot exceed the degree of the antecedent warrant for $\mathrm{Q}$.

The example in $\S 2$ is such that (to simplify let us disregard again P-WARRANT and DEPENDENCE):

(b) The warrant from $\mathrm{E}$ for $\mathrm{P}$ is not counterfactually independent of the antecedently warrant for Q AND the degree of the new warrant for $\mathrm{Q}$ does exceed the degree of the antecedent warrant for $\mathrm{Q}$.

(a) and (b) appear compatible because (a)'s antecedent can be false when (b)'s left conjoint is true. The antecedent of (a) says that if $Q$ were not antecedently warranted, E would not warrant $P$ (which means that in all closest possible worlds where $\mathrm{Q}$ is not antecedently warranted, $\mathrm{E}$ does not warrant $\mathrm{P}$ ). The left conjoint of (b) says that it is false that if $\mathrm{Q}$ were not antecedently warranted, E would warrant $\mathrm{P}$ (which means that in some of the closest possible worlds where $\mathrm{Q}$ is not antecedently warranted, E does not warrant $\mathrm{P}$ ). 
$\operatorname{Pr}_{\mathrm{E}}(\mathrm{P})=1 / 6<t-$ i.e. $\mathrm{E}$ does not warrant P. Since in the closest possible worlds where FAIL1's antecedent is true its consequent is true, FAIL1 is true in Balls.

Our interim conclusion is that FAIL1 is not satisfactory because it does not block transmission of intensifying warrant. Note that our counterexample does not depend on selecting just 3/4 as the threshold value $t$ for warranted belief. Any other $t \in[1 / 2,3 / 4)$ would also do. Furthermore, Balls could be modified to satisfy any higher $t \in[3 / 4,1)$ one might propose. We return to the problem of intensifying warrant in the next section.

For the sake of completeness we need to touch upon another issue before concluding the section. Wright (2002) proposes - or at least presupposes - a second condition sufficient for transmission failure, which appears less problematic but more trivial than the former. To isolate this condition we need to refer to Wright's notion of supplying a direct warrant for a proposition. This is what Wright (ibid.: 334) substantially says: if P entails Q, the warrant afforded by E for Q is direct relative to $\mathrm{P}$ if and only if $\mathrm{E}$ would have warranted $\mathrm{Q}$ even if $\mathrm{E}$ had not warranted $\mathrm{P}$. Here is an example (ibid.: 333):

(Soccer)

E. Jones has just kicked the ball between the white posts.

P. Jones has just scored a goal.

Therefore:

Q. A game of soccer is taking place.

Although E warrants $\mathrm{P}$ and $\mathrm{P}$ is known to entail Q (given ordinary background information), E affords just a direct warrant for Q relative to P. For E would warrant Q even if E no longer warranted P. To see this, think of a situation slightly different from the (imagined) actual in which, just before seeing Jones kicking the ball between the white posts, you had noticed that the referee's assistant raised her flag to signal Jones' off-side. This implies that your counterfactual background information at the time at which you acquires E slightly differs from your actual 
background information at that time. Against this altered background information, E no longer warrants P but still warrants Q.

According to Wright, if $\mathrm{P}$ entails $\mathrm{Q}$, and $\mathrm{E}$ provides a warrant for $\mathrm{P}$ and only a direct warrant for $\mathrm{Q}$ relative to $\mathrm{P}$, it is intuitively true that the argument from $\mathrm{P}$ to $\mathrm{Q}$ is not transmissive - thus Soccer is not transmissive. The intuition is that if $\mathrm{E}$ directly warrants $\mathrm{Q}$ relative to $\mathrm{P}$, the warrant acquired for $\mathrm{Q}$ is unmediated by $\mathrm{P}$. Thus this warrant should not "be regarded as transmitted via the inference" from P (Wright 2002: 334). Though in Soccer the direct warrant for Q relative to P is a first-time warrant, there may be cases in which the direct warrant for Q relative to $\mathrm{P}$ is a new warrant. $^{28}$

Wright's second sufficient condition for transmission failure can be formulated as follows:

(FAIL2) E supplies Q with just a direct warrant relative to $\mathrm{P}$.

This condition fits the account of warrant transmission presented in $\S 2$. For if FAIL2 is true, DEPENDENCE is false, with the consequence that warrant transmission - any type of warrant transmission - fails. This is so because DEPENDENCE says, among other things, that there would be no first-time warrant (if Q is not antecedently warranted) or no new warrant for Q (if Q is antecedently warranted), if E did not supply a warrant for P. But if FAIL2 is true, this is false, because E supplies a first-time warrant or a new warrant for Q that would survive even if E did not warrant $\mathrm{P}^{29}$

\section{Intensifying warrant: a recalcitrant case?}

\footnotetext{
${ }^{28}$ Consider for instance a variant of Soccer in which your background information alone gives you a warrant for Q (e.g. you are told that Q). In this case E could give you a new direct warrant for Q.

${ }^{29}$ Since we concentrate on evidential warrant (see note 4 above), we leave out the explanation of failure of transmission of non-evidential warrant worked out by Wright especially in relation to his analysis of the McKinsey paradox (see for instance 2011).
} 
We have suggested that FAIL1 gives a natural and apparently plausible interpretation of Wright's more interesting condition for transmission failure. We have also shown that, despite this, FAIL1 does not square with the analysis of warrant transmission offered in $\S 2$. For Balls appears to satisfy FAIL1 together with P-WARRANT, DEPENDENCE and Q-W3. So FAIL1 is incapable to block transmission of intensifying warrant, while Wright seems to take his condition to be capable to block warrant transmission across the board. This problem lends itself to at least three different lines of response. The one we find more natural consists of two steps: first, acknowledging that FAIL1 substantially coincides with Wright's condition for transmission failure and that this condition is defective in the sense indicated; second, producing an expansion of FAIL1 capable to block transmission of intensifying warrant. We call this line of response ampliative. Alternatively, one could try to resist the Balls objection. A first way to go is simply rejecting the Bayesian formalization presupposed in Balls as problematic or misleading. We call this strategy conservative because it does not question the assumption that FAIL1 is a sufficient condition for transmission failure in general. A subtler way to resist Balls is denying that FAIL1 accurately reproduces Wright's condition for transmission failure - we call this reply attuning because it imposes adjustments on FAIL1. This strategy aims to show that a more fitting version of FAIL1 is not satisfied in Balls. Let us focus on each of these strategies in turn.

Ampliative strategy. The most elementary expansion of FAIL1 capable to block transmission of intensifying warrant is this:

(FAIL1*) If there were no antecedent warrant for Q, E would not supply a warrant for P, and $\left(^{*}\right)$ if there were antecedent warrant for $Q$, there would be no new warrant for Q stronger than Q's antecedent warrant. 
The additional clause (*) is overtly incompatible with Q-W3. ${ }^{30}$ A reason of discontent with (*) is that it is often not immediately clear how one could ascertain its truth-value. To settle this snag we suggest to reformulate $(*)$ as follows:

(**) If there were antecedent warrant for $\mathrm{Q}, \mathrm{E}$ would be positively relevant or irrelevant to $\sim Q .{ }^{31}$

By saying that $\mathrm{E}$ is positively relevant to $\sim \mathrm{Q}$ we mean that $\mathrm{E}$ increases the degree of warrant antecedently available for $\sim \mathrm{Q}$. By saying that $\mathrm{E}$ is irrelevant to $\sim \mathrm{Q}$ we mean that $\mathrm{E}$ leaves the degree of warrant antecedently available for $\sim \mathrm{Q}$ unchanged. Note that $\mathrm{E}$ is positively relevant to $\sim \mathrm{Q}$ if and only if $\mathrm{E}$ is negatively relevant to $\mathrm{Q}-\mathrm{i}$.e. if and only if $\mathrm{E}$ decreases the degree of warrant antecedently available for $\mathrm{Q}$. Note also that $\mathrm{E}$ is irrelevant to $\sim \mathrm{Q}$ if and only if $\mathrm{E}$ is irrelevant to $\mathrm{Q}$. In conclusion, if $\mathrm{E}$ is positively relevant or irrelevant to $\sim \mathrm{Q}$, when $\mathrm{E}$ is acquired, the antecedent degree of Q's warrant cannot increase. So if $(* *)$ is fulfilled, there cannot be transmission of intensifying warrant.

Though $(* *)$ is equivalent to $(*),{ }^{32}$ the first formulation is more helpful than the second because its truth-value is often easier to ascertain. Consider again the paradigmatic case of transmission failure examined in $\S 4$. Suppose background information says that Jessica and Jocelyn are indistinguishable twins and consider again the following reasoning:

(Twins)

E. This girl looks just like Jessica.

P. This girl is actually Jessica.

Therefore:

Q. This girl is not Jocelyn.

\footnotetext{
${ }^{30} \mathrm{Q}-\mathrm{W} 3$ states that Q is antecedently warranted and there is a new warrant for Q stronger than Q's antecedent warrant, whereas (*) entails that if Q is antecedently warranted, there is no new warrant for Q stronger than Q's antecedent warrant.

${ }^{31}$ Where $\sim$ is logical negation.

${ }^{32}$ Tucker (2010) contends that the ground of Q's warrant in case of transmission is, not E, but P. Tucker must mean that the ground of Q's warrant is $\mathrm{P}$ only once $P$ has been in turn warranted by E. Since the ultimate source of warrant for $\mathrm{Q}$ would be $\mathrm{E}$, it would seem to be intuitive that if $\mathrm{E}$ alone does not increase the warrant for $\mathrm{Q}$ then $\mathrm{E}$ and $\mathrm{P}$ together do not increase the warrant for $Q$, and vice versa.
} 
Consider now the closest possible worlds in which Q is antecedently warranted. In these worlds, if $\mathrm{Q}$ is not certain, since Jessica and Jocelyn are indistinguishable, E is positively relevant to both $\mathrm{P}$ and $\sim$ Q (i.e. "This girl is actually Jocelyn"). So E must be negatively relevant to Q. On the other hand, if Q is just certain, E can only be irrelevant to both $\sim \mathrm{Q}$ and Q. Since (**) is satisfied, no intensifying warrant can transmit in Twins.

Those who opt for the ampliative strategy could thus replace FAIL1 with:

(FAIL3) If there were no antecedent warrant for Q, E would not supply a warrant for P, and if there were antecedent warrant for Q, E would be positively relevant or irrelevant to $\sim \mathrm{Q}$.

FAIL3 explains why intensifying warrant does not transmit in Twins and also in philosophically more interesting cases - e.g. in Dretske's famous zebra argument. ${ }^{33}$ Note, on the other hand, that FAIL3 is not satisfied in Balls because E ("The ball extracted ball is heavy") is negatively relevant to $\sim \mathrm{Q}$ ("The extracted ball is white"). This allows intensifying warrant to transmit.

Conservative strategy. Those unsympathetic towards formalizations could try to resist the Balls objection by disallowing the Bayesian model underlying Balls. One could perhaps question the assumption that belief in a proposition $\mathrm{X}$ is warranted just in case $\mathrm{X}$ 's subjective probability exceeds a precise threshold value. It might be adduced, for example, that elementary variants of the lottery and the preface paradox stem from this assumption. ${ }^{34}$ One could also dispute the assumption underlying Balls that increasing X's probability results in increasing X's degree of warrant by claiming, perhaps, that it is dubious that an increase of subjective probability always results in an increase of degree of warrant. In these ways one could try to challenge the thesis that

\footnotetext{
${ }^{33}$ In zebra, $\mathrm{E}=$ "The animal in the pen looks like a zebra", $\mathrm{P}=$ "The animal in the pen is a zebra" and $\mathrm{Q}=$ "The animal in the pen is not a cleverly disguised mule". Here E is clearly positively relevant to $\sim Q$ ("The animal in the pen is a cleverly disguised mule").

34 The lottery and the preface paradox can be reformulated to apply to warranted belief (rather than rational belief). The standard formulations of the two paradoxes can be found for instance in Sturgeon (2008).
} 
Q-W3 is satisfied in Balls. Particularly, one could try to dispute both the thesis that since $\operatorname{Pr}(\mathrm{Q})>$ $t$, $\mathrm{Q}$ is antecedently warranted, and the thesis that as $\operatorname{Pr}_{\mathrm{E}}(\mathrm{Q})>\operatorname{Pr}(\mathrm{Q})$, there is an intensifying warrant for Q.

This conservative strategy does not look promising to us. For we believe that the strength of the objection resting on Balls is largely independent of the Bayesian model, which essentially serves to simplify the discussion. For instance, we may concede that giving a precise value to the threshold for warranted belief is impossible without dropping the claim that Q proves warranted as one is told that box $C$ has been selected. This claim is independently plausible: $\operatorname{since} \operatorname{Pr}(\mathrm{Q})=$ $4 / 5$, it is hard to see how one could deny that Q is warranted. Note also that Balls can easily be modified to arbitrarily increase the value of $\operatorname{Pr}(\mathrm{Q})$ to approximate to 1 . We may also grant that an increase of subjective probability not always results in an increase of degree of warrant and yet still contend that, when box $C$ is selected, the antecedent warrant for $\mathrm{Q}$ is strengthened. It seems intuitive (at least to us) that in Balls the warrant for Q increases as E is learned. Note also that Balls could easily be modified by arbitrarily raising $\operatorname{Pr}_{\mathrm{E}}(\mathrm{Q})$ up to $1 .^{35,36}$

Attuning strategy. One could concede that FAIL1 is satisfied in Balls while disputing that FAIL1 accurately reflects Wright's condition for transmission failure. The contention would be that our proposed reading of Wright's condition - encoded by FAIL1 - is uncharitable and should be replaced by a more suitable interpretation. One could try to argue for instance along these lines: Wright claims that the warrant from E for P fails to transmit to P's consequence Q whenever the warrant from E for P depends on antecedent warrant for Q. FAIL1 does not to fully capture

\footnotetext{
${ }^{35}$ If $\operatorname{Pr}_{\mathrm{E}}(\mathrm{Q})=1$, E makes Q certain. Before E was acquired, Q was uncertain. It is hard to see how one could deny that at least in this case the warrant for $\mathrm{Q}$ has been strengthened.

${ }^{36}$ The conservative strategy looks even less promising if the target is, not Q-W3, but FAIL1, DEPENDENCE or PWARRANT - i.e. if it is used to show that the latter three conditions are unsatisfied in Balls. For instance, we have claimed that FAIL1 appears satisfied because in the closest possible worlds where Q is not antecedently warranted i.e. where box $A$ has been randomly selected $-\mathrm{E}$ does not supply a warrant for $\mathrm{P}$. These are worlds in which $\operatorname{Pr}(\mathrm{Q})=$ $1 / 2$ and $\operatorname{Pr}_{\mathrm{E}}(\mathrm{P})=1 / 6$. It is hard to question our claim because it is very intuitive that if $\operatorname{Pr}(\mathrm{Q})=1 / 2$, belief in $\mathrm{Q}$ is not antecedently warranted, and that if $\operatorname{Pr}_{\mathrm{E}}(\mathrm{P})=1 / 6$, E does not warrant $\mathrm{P}$.
} 
Wright's claim because the notion of dependence that Wright presupposes is more sophisticated than the one reflected by FAIL1. One might suggest that Wright requires the dependence at issue to be in some sense basic or fundamental.

A way to understand the notion of fundamental dependence could be the following. Suppose X, $\mathrm{Y}$ and $\mathrm{Z}$ are distinct propositions or states of affairs. Let us say that $\mathrm{X}$ depends on $\mathrm{Y}$ more fundamentally than it depends on $\mathrm{Z}$ just in case the following conditions are fulfilled:

(i) If it were not the case that $Y$, it would not be the case that $\mathrm{X}$.

(ii) If it were not the case that $\mathrm{Z}$, it would not be the case that $\mathrm{X}$.

(iii) If it were not the case that $\mathrm{Z}$ and it were the case that $\mathrm{Y}$, it would be the case that $\mathrm{X}$.

(iv) If it were not the case that $\mathrm{Y}$ and it were the case that $\mathrm{Z}$, it would not be the case that $\mathrm{X}$.

Clauses (i) and (ii) say that X depends on, respectively, Y and Z. Clauses (iii) and (iv) specify that $\mathrm{X}$ depends on Y more fundamentally than it depends on Z. Once this account is in place, the notion of fundamental dependence can be defined as follows: $\mathrm{X}$ fundamentally depends on $\mathrm{Z}$ if and only if (ii) if it were not the case that $\mathrm{Z}$ it would not be the case that $\mathrm{X}$, and there is no $\mathrm{Y}$ such that $\mathrm{X}$ depends on Y more fundamentally than it depends on Z. Namely, there is no Y such that (i), (iii) and (iv) are also jointly satisfied. ${ }^{37} \mathrm{~A}$ consequence of this definition is that $\mathrm{X}$ does not fundamentally depend on $\mathrm{Z}$ if there is a $\mathrm{Y}$ on which $\mathrm{X}$ more fundamentally depends. One might contend that FAIL1 should be replaced by this condition:

(FAIL4) The state of affair that E supplies a warrant for P fundamentally depends on the state of affair that $\mathrm{Q}$ is antecedently warranted.

Since FAIL4 entails FAIL1, satisfying FAIL4 prevents the satisfaction of Q-W1 and Q-W2. Furthermore, once FAIL4 is in place, Balls does not threaten Wright's analysis of transmission

\footnotetext{
${ }^{37}$ Note that this definition does not preclude the possibility that X fundamentally depends on distinct sates of affairs all at once (e.g. on $\mathrm{Z}$ and on $\mathrm{Y}$ ).
} 
failure any longer because FAIL4 is not satisfied in Balls. We have seen that in Balls the state of affair (X) that E supplies a warrant for $\mathrm{P}$ depends on the state of affair $(\mathrm{Z})$ that $\mathrm{Q}$ is antecedently warranted in the sense of (ii): if it were not the case that Z, it would not be the case that X. (This is why FAIL1 is satisfied). Yet this dependence is not fundamental. For X depends, in Balls, more fundamentally on the state of affairs (Y) that your background information says that the ratio of the red and heavy balls to the heavy balls is $5 / 6$.

We saw that $\mathrm{X}$ and $\mathrm{Z}$ do satisfy (ii). Let us now show that $\mathrm{X}, \mathrm{Y}$ and $\mathrm{Z}$ also satisfy (i), (iii) and (iv), to the effect that $\mathrm{X}$ depends more fundamentally on $\mathrm{Y}$ than it depends on $\mathrm{Z}$. Consider first that if $\mathrm{Y}$ had not been the case, $\mathrm{X}$ would not have been the case - i.e. if your background information had not included the belief that the ratio of the red and heavy balls to the heavy balls is 5/6, E would not have warranted your belief in P. This would have happened, intuitively, if you had been told that you have randomly selected box $A$ or $B$ rather than $C$. In that case you would have believed that the ratio of the red and heavy balls to the heavy balls is respectively $1 / 6$ or $1 / 2$. In this case, learning E would not have warranted your belief in $\mathrm{P}$, as it would have turned out that, respectively, $\operatorname{Pr}_{\mathrm{E}}(\mathrm{P})=1 / 6<t$ or $\operatorname{Pr}_{\mathrm{E}}(\mathrm{P})=1 / 2<t$. Hence (i) is fulfilled. Consider now that if $\mathrm{Z}$ had not been the case and $\mathrm{Y}$ had been the case, $\mathrm{X}$ would have been the case. The closest possible worlds in which Q is not warranted but you still believe that the ratio of the red and heavy balls to the heavy balls is $5 / 6$ are presumably those in which you are still told you have selected box $C$ and the only changes depend on the fact that your initial information does not tell the number of black balls in $C$, so that $\mathrm{Q}$ is not antecedently warranted for you. In these closest worlds $\mathrm{E}$ gives you a warrant for $\mathrm{P}$ because $\operatorname{Pr}(\mathrm{P})=7 / 20<t$ while $\operatorname{Pr}_{\mathrm{E}}(\mathrm{P})=5 / 6>t$. Hence, if $\mathrm{Z}$ had not been the case and Y had been the case, $\mathrm{X}$ would have been the case. Thus (iii) is satisfied. Finally, consider that if $\mathrm{Y}$ had not been the case and $\mathrm{Z}$ had been the case, $\mathrm{X}$ would not have been the case. This would happen, intuitively, if you had been told that you have selected box $B$ rather than $C$. In that case 
you would have believed that the ratio of the red and heavy balls to the heavy balls is $1 / 2$ rather than 5/6 but Q would have still been antecedently warranted for you, as $\operatorname{Pr}(\mathrm{Q})=4 / 5>t$. In this case, learning E would not have warranted your belief in $\mathrm{P}$, as it would have turned out that $\operatorname{Pr}_{\mathrm{E}}(\mathrm{P})$ $=1 / 2<t$. Hence (iv) is satisfied too.

As FAIL4 is not fulfilled in Balls, the attuning strategy would give its proponents a way out from the Balls objection. ${ }^{38}$ Note however that Balls has served two distinct purposes: showing that Q-W2 and Q-W3 are independent (argued for in $§ 2$ ), and giving a counterexample to the claim that satisfying Wright's condition prevents the fulfilment of Q-W3 (presented in §4). The attuning strategy, if successful, just shows that Balls is no counterexample to the latter claim. It does not also show that Q-W2 and Q-W3 are not independent. Consequently, the proponent of the attuning strategy should still show - in order to fully vindicate Wright - that fulfilling Wright's condition does prevent the fulfilment of Q-W3. (As this does not follow from the mere fact that Wright's condition prevents the fulfilment of Q-W2, if Q-W2 and Q-W3 are independent). Our impression is that no reasonable reinterpretation of Wight's condition could succeed in this task. ${ }^{39}$

\section{Conclusions}

We have offered a comprehensive analysis of transmission and failure of transmission of warrant. Whereas the current literature mainly concentrates on transmission and failure of transmission of first-time warrant, we have aimed at characterising a broader class of phenomena encompassing

\footnotetext{
${ }^{38}$ It seems undeniable that FAIL4 is satisfied in Twins: the state of affair that E ("This girl looks just like Jessica") provides a warrant for P ("This girl is actually Jessica") fundamentally depends on the state of affairs that Q ("This girl is not Jocelyn") is antecedently warranted.

${ }^{39}$ Our scepticism is only motivated by the fact that Q-W3 and Q-W2 are independent. It is not motivated by the concern (which might have stroke some reader from the start) that any counterfactual account of the dependence relation is doomed to fail. It might well be that the project of accounting for the dependence relation in counterfactual terms is fraught with insurmountable difficulties. But as far as this separate issue is concerned, we prefer to retain a cautious optimism in favour of the counterfactual account. Our point is simply that since Q-W3 and Q-W2 are independent, no reinterpretation of Wright's condition - whether or not couched in counterfactuals terms - appears probable to prevent the satisfaction of Q-W2 and Q-W3 at once.
} 
the latter. We have singled out three conditions individually necessary and jointly sufficient for transmission of warrant. We have shown that their satisfaction grounds a variety of types of epistemic transmission of which of transmission of first-time warrant is just one case; other types include at least transmission of ground-extending warrant, proxy warrant, and intensifying warrant. Our analysis of the last type of transmission has turned out to lead to a potential disagreement with Wright. Wright has proposed one condition that he apparently considers to be sufficient for transmission failure across the board. Our Bayesian example suggests, however, that intensifying warrant can transmit even if Wright's condition is fulfilled. We have considered three possible lines of reply: accepting that Wright's condition is imperfect and working out a more inclusive condition for failure of transmission; dismissing the Bayesian counterexample by dropping its assumptions; working out a different, possibly more accurate interpretation of Wright's analysis capable to defuse the counterexample. We have suggested ways to develop each of these strategies; at the same time, however, we have given reasons for preferring the first.

Acknowledgments We are grateful to Greg Wheeler and two referees of this Journal for valuable feedback.

\section{References}

Chandler, J. (2010). 'The transmission of support: a Bayesian re-analysis'. Synthese 176: 333-343. Ebert. A. P. (2005). 'Transmission of Warrant-Failure and the Notion of Epistemic Analyticity'. Australasian Journal of Philosophy 83: 505-521.

Moretti, L. (2011). 'Wright, Okasha and Chandler on transmission failure' Synthese (forthcoming).

Okasha, S. (2004). 'Wright on the Transmission of Support: A Bayesian Analysis.' Analysis 64: 139-146. 
Silins, N. (2005). 'Transmission failure failure'. Philosophical Studies 126: 71-102.

Smith, M. (2009). 'Transmission failure explained'. Philosophy and Phenomenological Research 79: 164-189.

Sturgeon, S. (2008). 'Reason and the Grain of Belief'. Nous 42: 139-165.

Tucker, C. (2010). 'When Transmission Fails'. Philosophical Review 119: 497-529.

Williamson, T. (2000). Knowledge and its limits. Oxford: OUP.

Wright, C. (1985). 'Facts and Certainty'. Proceedings of the British Academy, 71: 429-472.

Wright, C. (2002). '(Anti-)Sceptics Simple and Subtle: G. E. Moore and John McDowell'. Philosophy and Phenomenological Research, 65: 330-348.

Wright, C. (2003). 'Some Reflections on the Acquisition of Warrant by Inference'. In S. Nuccetelli (Ed.), New Essays on Semantic Externalism and Self-Knowledge, pp. 57-77. Cambridge, Mass.: MIT Press.

Wright, C. (2004). 'Warrant for Nothing (and Foundations for Free?)'. Proceedings of the Aristotelian Society, Supplementary Volume, 78: 167-212.

Wright, C. (2007). 'Perils of Dogmatism'. In S. Nuccetelli (Ed.), Themes from G. E. Moore: New Essays in Epistemology and Ethics, pp. 25-48. Oxford: OUP.

Wright, C. (2011). 'McKinsey One More Time'. In A. Hatzimoysis (Ed.), Self-Knowledge, pp. 80104. Oxford: OUP. 